\title{
Der thermische Zerfall des Chlorhexoxyds in Gegenwart von Fluor
}

\author{
Von A. J. Arvia, W. H. Basualdo ${ }^{1}$ ) und H. J. Schumacher
}

Mit 3 Abbildungen

\section{Inhaltsübersicht}

Es wird der thermische Zerfall des Chlorhexoxyds in Gegenwart von Fluor innerhalb eines Druckgebietes von $40-375 \mathrm{~mm} \mathrm{Hg}$ bei Temperaturen zwischen 22 und $48^{\circ} \mathrm{C}$ untersucht.

Als Reaktionsprodukte entstehen etwa $70-75 \%$ Chlorylfluorid, etwa $25 \%$ Chlorheptoxyd, einige Prozent Chlor, geringe Mengen einer flüchtigen, Fluor, Chlor und Sauerstoff enthaltenden Verbindung, die nicht mit Sicherheit identifiziert werden konnte, aber möglicherweise $\mathrm{FClO}_{4}$ ist, und die entsprechenden Mengen an Sauerstoff.

Weder das Hexoxyd noch das Trioxyd reagieren mit Fluor. Das Trioxyd geht verschiedene Zerfallsreaktionen ein. Eine führt zur Bildung von Chlordioxyd und Sauerstoff, eine weitere $\mathrm{zu}$ der von $\mathrm{ClO}_{4}$ und Chlordioxyd und eine dritte zu Chlor und Sauerstoff. Die Aktivierungsenergien dieser 3 Reaktionen weichen nicht sehr stark voneinander ab. Sie betragen etwa $12 \pm 2 \mathrm{kcal}$.

Der überwiegende Teil des Chlordioxyds wird durch Realtion mit Fluor zu Chlorylfluorid umgesetzt, während die $\mathrm{ClO}_{4}$-Radikale mit $\mathrm{ClO}_{3}$ Chlorheptoxyd bilden.

Es gelingt mit Hilfe der neuen Erkenntnisse, bisher ungeklärte Beobachtungen beim durch Chlor sensibilisierten thermischen Ozonzerfall zu erklären und für diese Reaktion ein vollständiges Reaktionsschema anzugeben. Ebenso konnten einige experimentelle Ergebnisse von Rolrefson und von Gooneve und Mitarbeitern, die die thermische Reaktion zwischen Chlortrioxyd und Ozon und die photochemische Reaktion zwischen Chlor und Ozon bzw. den thermischen Zerfall des Chlorhexoxyds untersuchten, gedeutet werden.

Im Laufe von systematischen Studien über die Reaktionen der Halogene mit anorganischen Verbindungen untersuchten wir auch die Reaktion zwischen Fluor und Chlorhexoxyd. Wir wählten diese Reaktion aus, weil wir es für möglich hielten, hier eine Reaktion vor uns zu haben, die in einfacher Weise zur Bildung des Perchlorylfluorids $\left.\left(\mathrm{FClO}_{3}\right)^{2}\right)$ oder seiner isomeren Verbindung, des Chloryloxyfluorids

1) Die ausführlichen Versuche sind in der Dissertation von W. H. Basualdo, Fac. de Quím. y Farm., Univ. de La Plata, wiedergegeben.

2) A. Engelbrecht u. H. Atgwanger, Mh. Chem. 83, 1087 (1952). 
$\left.\left.\left.\left(\mathrm{ClO}_{2}-\mathrm{OF}\right)^{3}\right)^{4}\right)^{5}\right)$, führte. Wir wurden zu dieser Annahme vor allem dadurch geführt, daß sich das Chlorylfluorid $\left.\left.\left(\mathrm{FClO}_{2}\right)^{6}\right)^{7}\right)^{8}$ ), quantitativ in direkter Reaktion zwischen Fluor und Chlordioxyd bildet.

Wir hofften außerdem im Laufe dieser Untersuchungen unsere Kenntnisse über das chemische Verhalten des Chlorhexoxyds und des Chlortrioxyds zu vertiefen, von Verbindungen, die bei einigen Reaktionen, wo sie als Zwischenprodukte auftreten, eine erhębliche Rolle spielen. Wir wollen hier nur den durch Chlor sensibilisierten Ozonzerfall $\left.^{9}\right)^{10}$ ), die photochemische Reaktion zwischen Chlor und Ozon ${ }^{11}$ ) ${ }^{12}$ ) und die thermische Reaktion zwischen Chlortrioxyd und Ozon ${ }^{13}$ ) erwähnen. Bei der Deutung der bei diesen Reaktionen auftretenden Erscheinungen waren noch eine Reihe von Fragen offen geblieben, von denen zu vermuten war, daß sie bei Vorhandensein besserer Kenntnisse über das Hexoxyd geklärt werden konnten.

Unsere Versuche zeigten an, daß das Chlorhexoxyd und auch das Chlortrioxyd selbst nicht mit Fluor reagieren, wohl aber die bei ihrem thermischen Zerfall auftretenden Zwischenprodukte. Hierdurch wird es ermöglicht, ,stabile“ Verhältnisse zu schaffen und den Mechanismus des thermischen Zerfalls von Chlorhexoxyd aufzuklären.

Bei der im folgenden näher beschriebenen Untersuchung handelt es sich infolgedessen eigentlich nicht mehr um das Studium der Reaktion zwischen Chlorhexoxyd und Fluor als vielmehr um das des thermischen Zerfalls des Chlorhexoxyds in Gegenwart von Fluor.

\section{Die Apparatur}

Die Reaktion sollte in der Weise untersucht werden, daß Fluor von variablem aber bekanntem Druck auf bestimmte Mengen von Chlor-

3) H. Bode u. E. Klesper, Z. anorg. allg. Chem. 265, 275 (1951).

$\left.{ }^{4}\right)$ A. Engelbrecht, Angew. Chem. 66, 442 (1954).

5) H. Bode u. E. Klesper, Angew. Chem. 66, 605 (1954).

6) H. Schmitz u. H. J. Schumacher, Z. anorg. allg. Chem. 249, 238 (1942).

7) P. J. Aymonino, J. E. Sicre u. H. J. Schumacher, J. chem. Physics 22, 756 (1954).

8) P. J. Aymonino, J. E. Sicre u. H. J. Schumacher, An. Asoe. quím. argent. 43, 26 (1955).

9) M. Bodenstein, E. Padelt u. H. J. Schumacher, Z. physik. Chem., Abt. B 5, 209 (1929).

10) A. Hamann u. H. J. Schumacher, Z. physik. Chem., Abt. B 17, 293 (1932).

11) A. J. Allmand u. J. W. T. Spinks, J. chem. Soc. [London] 1931, 1652.

12) L. J. Heidt, G. B. Kistiakowsky u. G. S. Forbes, J. Amer. chem. Soc. 55, 223 (1933).

13) G. K. Rollefson u. A. C. Brrns, J. Amer. chem. Soc. 56, 364 (1934). 
hexoxyd, das zu jedem Versuch frisch im Reaktionsgefä $ß$ hergestellt war, bei konstanter Temperatur zur Einwirkung gelangte. Es wurde diese Form der Herstellung des Hexoxydes gewählt, um seine jeweils zur Reaktion gelangende Menge im voraus mit ausreichender Genauigkeit bestimmen zu können und um sicher zu sein, stets ein reines Produkt vor sich zu haben.

Da das Chlorhexoxyd bei den Reaktionstemperaturen $\left(22-48^{\circ} \mathrm{C}\right)$ eine viskose Flüssigkeit von relativ geringem Dampfdruck darstellt, $\left.\left(\mathrm{p}_{\mathrm{Cl}_{2} \mathrm{O}_{8} 0^{\circ} \mathrm{C}}=0,31 \mathrm{~mm} \mathrm{Hg} ; \mathrm{p}_{\mathrm{Cl}_{2} \mathrm{O}_{5} 48^{\circ} \mathrm{O}}=4,1 \mathrm{~mm} \mathrm{Hg}\right)^{14}\right)$, die zu erwartenden Produkte aber leicht flüchtig sind, so sollte der Reaktionsverlauf durch Druckmessungen verfolgt werden. Es war ferner beabsichtigt, die Identifizierung der Produkte durch Dampfdruckmessungen und durch chemische Analyse von dem Reaktionsgemisch entnommenen Proben vorzunehmen.

Die ersten Versuche wurden in ein€m Reaktionsgefä $@$ aus Pyrex-Glas durchgeführt und die Drucke an einem offenen mit konz. Schwefelsäure überdeckten Quecksilbermanometer gemessen. Es zeigte sich jedoch, daß sich unter diesen Bedingungen neben

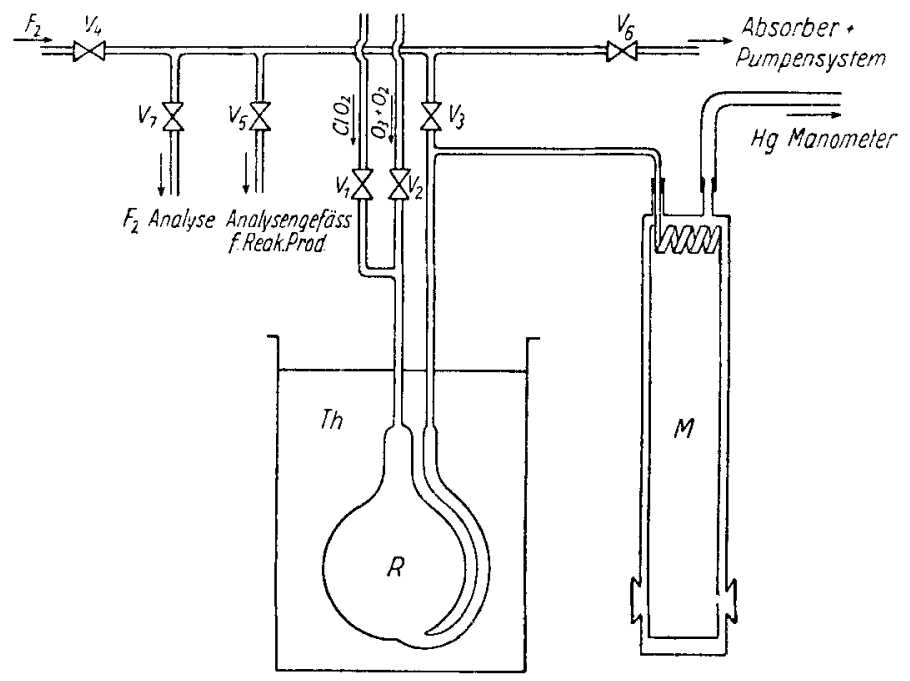

Abb. 1. Skizze der Apparatur

den eigentlichen Produkten stets hellrot bis dunkelrot gefärbte Substanzen bildeten, die, obwohl mengenmäßig wenig bedeutend, doch das eigentliche Reaktionsbild verschleierten und die quantitative Untersuchung der Reaktion stark behinderten. Die Bildung dieser Produkte war durch das Glas und die Schwefelsäure bedingt und blieb

14) C. F. Goodeve u. F. D. Richardson, J. chem. Soc. [London] 1937, 294. 
erst aus, als das gesamte Reaktionssystem aus Quarz erstellt und als Manometer ein Quarzspiralmanometer nach BoDENSTEIN, das als Nullinstrument diente, verwendet wurde. An Stelle von Hähnen wurden Aluminiumventile ${ }^{15}$ ) mit Teflondichtung benutzt. Diese Ventile, die einen stumpfen Konus aus Nickel und einen Sitz aus Teflon besaßen, bewährten sich sehr. Sie wurden weder von Ozon noch von den verschiedenen Chloroxyden angegriffen und konnten leicht feinreguliert werden.

In $\mathrm{Abb}, 1$ ist eine schematische Skizze der Apparatur wiedergegeben. Das ReaktionsgefäB R, aus durchsichtigem Quarz mit einem Volumen von $330 \mathrm{~cm}^{3}$ befand sich in dem Wasserthermostaten, Th. Das obere Ende von $R$ war über zwei Kapillaren mit den Ventilen $V_{1}$ und $V_{2}$ verbunden, die zur Chlordioxyd- und zur Ozonleitung führten; das untere Ende von $R$ war durch ein Rohr, das in eine Kapillare endete, mit dem BonensternManometer $M$ und über das Ventil $V_{s}$ mit der übrigen Apparatur - dem Pumpsystem, den Absorbern, der Fluorleitung und dem Analysensystem - verbunden.

\section{Die Herstellung und Reinigung der Ausgangsstoffe}

a) Chlordioxyd wurde nach der Methode von BRAY $\left.{ }^{16}\right)$, Schmiot ${ }^{17}$ ) und Mitarbeitern durch Einwirkung von $2 / 3$ n Schwefelsäure auf ei Gemisch von Kaliumchlorat und Oxalsänre hergestellt und durch fraktionierte Destillation bei tiefer Temperatur gereinigt. Es wurde in einer Spiralfalle, mit fester Kohlensäure gelsühlt, aufbewahrt.

b) Ozon wurde, wie üblich, im Siemens-Ozonizator durch die Einwirkung stiller elektrischer Entladungen auf reinen, trockenen, elektrolytisch hergestellten Sauerstoff, hergestellt. Der Ozongehalt des Ozon-Sauerstoff-Gemisches betrug etwa 11\%.

c) Chlorhexoxyd wurde nach dem von SCHUMACHER und STIEG ER ${ }^{1 s}$ ) entwickelten Verfahren durch Reaktion von überschüssigem Ozon mit verdünntem Chlordioxyd gewonnen. Um jeweils eine bestimmte Menge von Chlorhexoxyd zu erhalten, wurde wie folgt gearbeitet: Ein konstanter Strom von trockenem Stickstoff strömte mit einer Geschwindigkeit von etwa $1,5 \mathrm{l} / \mathrm{Std}$. durch das auf $-43^{\circ} \mathrm{C}$ gekühlte und sich in einer Spiralfalle befindende Chlordioxyd. Um sicher zu sein, $\mathrm{da} \beta$ der Gasstrom ein bekannte Menge an Chlordioxyd enthielt, wurde der Gasstrom durch eine weitere, etwas tiefer gekühlte Falle geleitet, in der sich das Chlordioxyd in geringem Maße kondensierte. Die Temperatur $\left(-45^{\circ} \mathrm{C}\right)$ dieser letzteren Falle diente zur Berechnung des Chlordioxydpartialdruckes im Gasstrom, der durch das Ventil $V_{1}$ in das bereits mit ozonisiertem Sauerstoff gefüllte und auf $-10^{\circ} \mathrm{C}$ abgekühlte Reaktionsgefä $\mathrm{R}$ eintrat. Das Ozon-SauerstoffGemisch strömte durch $V_{2}$ ein (Geschwindigkeit etwa $5 \mathrm{I} /$ Std.). Um sicher zu sein, da 3 auch alles Chlordioxyd während seiner Verweilzeit in $R$ umgesetzt war, wurde stets mit einem erheblichen Überschuß von Ozon gearbeitet. Waren die abziehenden Gase farblos, so war dies Ziel erreicht.

War die gew ünschte Menge von Hexoxyd kondensiert, so wurde der Chlordioxydstrom abgestellt, $V_{1}$ geschlossen, noch einige Minuten Ozon, dann reiner Sauerstoff durchgeleitet und schließlich, um etwa noch gelöstes Chlordioxyd zu entfernen, $\mathrm{R}$ auf $-30^{\circ} \mathrm{C}$ abgekühlt ·und mit der Diffusionspumpe evakuiert.

15) Näheres Dissertation von W. H. Basualdo.

$\left.{ }^{16}\right)$ W. BraY, Z. physik. Chem. 54, 575 (1906).

17) E. Schmist, E. Geisler, E. Arndt u. P. Ihlow, Ber. dtsch. chem. Ges. 56. 24 (1923).

1z) H. J. Schumacher u. G. Stieger, Z. anorg. allg. Chem. 184, 272 (1929). 
Das so hergestellte Chlorhexoxyd war, wie Analysen zeigten, weitgehend rein und enthielt vor allem keine nachweißbare Menge von Chlorheptoxyd. Seine Menge stimmte innerhalb von etwa $10 \%$ mit dem aus dem der Temperatur der Falle $\mathrm{F}_{2}$ entsprechenden Dampfdruck des Chlordioxyds und der Strömungsgeschwindigkeit des Stickstoffs berechneten Werte überein.

d) Fluor wurde durch Elektrolyse von geschmolzenem Kaliumbifluorid in einem Kupferelektrolyseur, der an anderer Stelle beschrieben ist ${ }^{8}$ ), hergestellt. Um störende Polarisationserscheinungen während der Elektrolyse zu vermeiden, waren dem Elektrolyten geringe Mengen (etwa 1\%) Lithiumfluorid zugegeben worden. Dies hatte auf die Stetigkeit der Elektrolyse einen sehr günstigen Einfluß.

Das Fluor wurde, um es von mitgerissenem Fluorwasserstoff zu befreien, durch einen Turm mit gekörntem, vorher geschmolzenem Natriumfluorid geleitet, passierte sodann, um alle kondensierbaren Verunreinigungen, im wesentlichen Kohlenstofffluoride, zu entfernen, eine mit flüssigem Sauerstoff gekühlte Kupferfalle und wurde schließlich in einem 31 großen Kupfergefäß aufbewahrt. Letzteres befand sich in einem Wasserthermostaten und war über ein Kupferventil mit einem empfindlichen Manometer verbunden.

Über das Aluminiumventil $\mathrm{V}_{4}$ konnte das Fluor in die eigentliche Apparatur eintreten. Wie Analysen ${ }^{19}$ ) zeigten, hatte es einen Reinheitsgrad, der stets zwischen 95 und $98 \% \mathrm{~F}_{2}$ lag.

\section{Die Durchführung der Versuche}

Die Versuche wurden derart durchgeführt, daß, nachdem die gewünschte Menge von $\mathrm{Hexoxyd}$ im Reaktionsgefä $B$ hergestellt war, dieses auf $0^{\circ} \mathrm{C}$ gebracht, die Drucke abgelesen und sodann die für den entsprechenden Versuch vorgesehene Menge Fluor eingelassen wurde. Vorversuche hatten ergeben, daß die Reaktion bei Temperaturen unterhaJb von $20^{\circ} \mathrm{C}$ nur sehr langsam verläuft, so daß also die eingelassene Fluormenge durch direkte Druckmessung bestimmt werden konnte. Wäre dies nicht möglich gewesen, so hät te man sie aus der Druckabnahme im Fluorgefäß und den bekannten Volumina dieses Vorratsgefäßes und des Reaktionsgefäßes berechnen müssen.

Das Reaktionsgefäß wurde sodann auf Versuchstemperatur gebracht und so lange auf dieser Temperatur belassen, bis das Hexoxyd verschwunden und, was gleichbedeutend hiermit war, keine weitere Druckänderung mehr auftrat. Die Reaktionsdauer betrug bei den angewendeten Hexoxydmengen und einer Temperatur von $40^{\circ} \mathrm{C}$ etwa $2-3$ Stunden; bei $25^{\circ} \mathrm{C}$ und sonst gleichen Bedingungen war sie etwa 7,5mal länger. Um die bei der Reaktion stattgefundene Druckzunahme zu bestimmen, wurde der Gesamtdruck bei $15^{\circ} \mathrm{C}$ gemessen. Diese Temperatur diente als Vergleichstemperatur, auf die alle bei anderen Temperaturen gemessenen Drucke umgerechnet wurden. $15^{\circ} \mathrm{C}$ wurde gewählt, weil bei tieferen Temperaturen mit der Kondensation von Reaktionsprodulaten gerechnet werden mußte. Bei den Umrechnungen wurden für den schädlichen Raum des Reaktions. systems, der stets auf Zimmertemperatur blieb, und der etwa 1\% des Gesamtvolumens ausmachte, in geeigneter Weise Korrekturen angebracht ebenso, wie bei den Druckangaben die beim Evakuieren im Reaktionsgefäß verbliebenen Restgase in entsprechender Weise berücksichtigt wurden.

Hierauf wurde $\mathrm{R}$ auf $-183^{\circ} \mathrm{C}$ gebracht und abermais der Druck gemessen. Von den in Frage stehenden Substanzen waren bei dieser Temperatur nur noch Fluor und Sauerstoff bzw. die inerten Beimischungen des Fluors als Gase vorhanden. Diese Messung ge-

19) H. Schmitz u. H. J. Schumacher, Z. anorg. allg. Chem. 945, 221 (1940). 
stattete also den während der Reaktion aufgetretenen Fluorverbrauch bzw. die Sauerstoffentwicklung festzustellen. Sodann wurde bei der gleichen tiefen Temperatur mit der Diffusionspumpe evakuiert und danach durch Erwärmen auf $20^{\circ} \mathrm{C}$ die Summe der kondensierbaren Reaktionsprodukte bestimmt.

Der Dampfdruck dieser Reaktionsprodukte wurde ebenfalls stets bei $-78^{\circ} \mathrm{C}$ gemessen. Da die Chloroxyde bei dieser Temperatur praktisch keinen Dampfdruck mehr haben, der des Chlorylfluorids bekannt ist $\left(8,8 \mathrm{~mm} \mathrm{Hg}\right.$ bei $\left.-78^{\circ} \mathrm{C}\right)$, so können aus der Höhe des gemessenen Druckes wichtige Schlüsse über die Art und Menge der nicht kondensierten Produkte gezogen werden.

Nach Abschluß der Druckmessungen wurden die Reaktionsprodukte über die Ventile $V_{3}$ und $V_{5}$ in drei Fraktionen nacheinander in das Analysengefä $B$ destilliert und nach dessen Abschmelzen analysiert.

Die erste Fraktion wurde bei $-108^{\circ} \mathrm{C}$ abdestilliert. Bei dieser Temperatur hat das Chlorylfluorid einem Dampfdruck von etwa 0,1 mm $\mathrm{Hg}$. Es können also nur Chlor, etwa vorhandenes Fluorperchlorat, $\mathrm{FClO}_{4}$, oder sonstige leichtflüchtige, nicht bekannte Fluorverbindungen übergehen. - Die zweite Destillation erfolgte bei $-70^{\circ} \mathrm{C}$. Bei dieser Temperatur hat das Chlorylfluorid einen Dampfdruck von etwa $17 \mathrm{~mm} \mathrm{Hg}$, während der Druck des Chlorheptoxydes - wie wir sehen werden, handelt es sich bei dem leichtkondensierbaren Reaktionsprodukte im diese Verbindung - nur etwa $0,1 \mathrm{~mm} \mathrm{Hg}$ beträgt. Um Úbersättigung in der Lösung, von der abdestilliert wurde, zu vermeiden, wurde die Destillation verschiedentlich unterbrochen, die Lösung aufgewärmt, dann wieder abgekühlt und mit der Destillation fortgefahren. - Die dritte Fraktion wurde bei Normaltemperatur destilliert. Es verblieb kein Rückstand.

Das Auffanggefä $B$, indem das Destillat kondensiert wurde und das auch als Analysengefäß diente, bestand aus Quarz. Es wurde mit flüssigem Sauerstoff gekühlt, so daß auf dieser Seite nur ein sehr kleiner Druck herrschte.

Bei der chemischen Analyse wurde stets, falls in alkalischer Lösung gearbeitet wurde, die Gesamtsäure- sowie der Gehalt an Hypochlorit-, Chlorit-, Chlorat- und Fluorionen bestimmt, während der Gehalt an Überchlorsäure nach der Differenzmethode berechnet wurde.

Fluor wurde maßanalytisch nach dem Verfahren von Armstrong ${ }^{20}$ ) und Rowley und CHuRCHuL ${ }^{21}$ ) bestimmt. Die in der Lösung vorhandenen Oxydationsmittel wurden mit $\mathrm{H}_{2} \mathrm{O}_{2}$ zerstört und dann $\mathrm{F}-$ bei Gegenwart von Natriumalizarinsulfonat mit Thoriumnitratlösung titriert.

Zur Bestimmung der verschiedenen Chlorsauerstoff-Säuren wurde nach einem im GMELIN ${ }^{2}$ ) angegebenen Verfahren vorgegangen. Der Gehalt an $\mathrm{ClO}^{-}$wurde in einem $\mathrm{NaHCO}_{3}$ enthaltenden Medium durch Zurücktitrieren des in der Lösung vorhandenen Überschusses an $\mathrm{H}_{3} \mathrm{AsO}_{3}$ mit $\mathrm{J}_{2}$ festgestellt, der an $\mathrm{ClO}_{2}^{-}$in der gleichen Lösung durch Titration des bei Gegenwart von saurer KJ-Lösung ausgeschiedenen $J_{2}$ mit $\mathrm{Na}_{2} \mathrm{~S}_{2} \mathrm{O}_{3}$ erhalten wurde. Die Menge an $\mathrm{ClO}_{3}^{-}$wurde nach einer von Treadwell-HaLL ${ }^{23}$ ) beschriebenen Methode bestimmt. Hierzu wurde in einem Teil der Probe der Gesamtoxydationswert festgestellt, indem in stark saurem Medium der Verbrauch von $\mathrm{Fe}^{2+}$ durch Rück-

20) W. D. Armstrong, Ind. Engng. Chem. analyt. Edit. 8, 384 (1936).

21) R. J. Rowley u. H. V. Churchild, Ind. Engng. Chem., analyt. Edit. 9, 551 (1937).

22) Gmelins Handbuch der anorg. Chem., 8. Aufl., System-Nummer 6, S. 307.

23) F. P. Treadwell u. W. T. Hall, Kurzes Lehrbuch der Analytischen Chemie, II B, Wien 1943, S. 542 . 
titation des Cberschusses mit $\mathrm{KMnO}_{4}$ gemessen wurde. Durch Jifferenzbildung dieses Wertes mit den Werten für den Gehalt an $\mathrm{ClO}^{-}$und $\mathrm{ClO}_{2}$, wurde dann der $\mathrm{ClO}_{3}$. Wert erhalten.

Bei der Analyse der Fraktion I wurde bisweilen auch mit saurer KJ-Lösung gearbeitet und dann das ausgeschiedene $J_{2}$ titricrt. Von den Fraktionen II und III, die sich als praktisch reine Produkte erwiesen, wurden einige Male die Dampfdruckkurven bestimmt. Diese Messungen wurden im Reaktionsgefä $B(R)$ vorgenommen und zu diesem Zwecke Fraktion III dort belassen bzw. Fraktion II nach R. nachdem dieses evakuicrt war, rückdestilliert.

\section{Die Ergebnisse}

Zunächst wurde beobachtet, daß die Reaktionsprodukte im gasförmigen Zustand farblos sind. Auch im ausgefrorenen Zustand waren die Fraktionen II und III farblos, während bei Fraktion I ein schwach gelblich gefärbter Ring auftrat, der offenbar aus $\mathrm{Cl}_{2}$ bestand.

Typisch war ferner, daß die 'Gesamtdruckzunahme in erster Anniherung mit dem Druck der kondensierbaren Reaktionsprodukte übereinstimmte, daß also der Druck der nicht kondensierbaren Gase im wesentlichen mit dern Anfangsdruck des Fluors und dessen inerten Beimengungen ïbercinstimmte.

Dies alles deutete bereits darauf hin, daß sich eine Verbindung vom Typ des $\mathrm{FClO}_{3}$ höchstens in sehr geringer Menge gebildet haben konnte und daß3 das in Fraktion II enthaltene Hauptprodukt Chlorylfluorid FClO ${ }_{2}$ sein mußte, dessen Bildung offenbar nach folgendem Schema erfolgt:

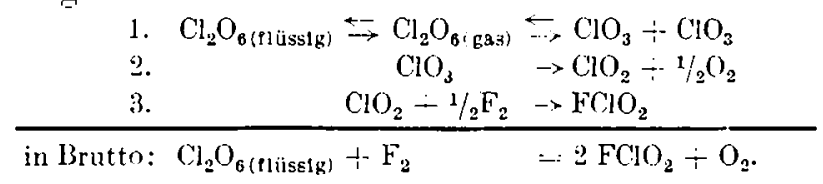

Der exakte beweis dafür, dals die zweite Fraktion aus reinem (hilorylfluorid bestand, wurde auf verschiedene Weise erbracht:

a) Die chemische Analyse ergab, was Säureverbrauch und Fluorgehalt anbelangt, in guter Annäherung die für $\mathrm{ClO}_{2} \mathrm{~F}$ zu erwartenden Werte, während vom Chlorat, das sich aus $\mathrm{ClO}_{2} \mathrm{~F}$ durch $\mathrm{Hydrolyse}$ bildet, durchweg $90-95 \%$ der berechncten Menge gefunden wurden. Der Rest fand sich als Hypochlorit und Chlorit. Der Grund für das Luftreten zu geringer Chloratwerte ist offenbar darin zu suchen, daß das Chlorylfluorid beim Zusammentreffen mit Wasser oder Wasserdampf stets gewisse Zersetzungsreaktionen eingeht, die nicht völlig auszuschalten sind.

b) Die Bestimmung der thermischen Stabilität der Substanz zeigte in Über'instimmung mit früheren Messungen ${ }^{24}$ ) am (hlorylfluorid, daB unterhalb von $200^{\circ} \mathrm{C}$ rin morklicher thermischer Zerfall nicht auf tritt.

() Die Dampfdruckwerte (Abb. 2) passen sich den Werten von Scumisz u. S(CH(:MACHER ${ }^{6}$ ) gut an.

24) IV. H. Basuali:o u. II. J. Schumar her, Angew. Chem. 67, 231 (1955). 
d) Auch das Verhalten der Substanz gegenüber Wasserdampf, Glas und Schwefelsäure, das stets zu Bildung rötlich gefärbter Substanzen führt, entspricht völlig dem des Chlorylfluorids.

Es kann hiermit als sicher angesehen werden, daß Fraktion II aus reinem Chlorylfluorid besteht. Unter unseren Versuchsbedingungen werden etwa $70-75 \%$ des als Ausgangsmaterial dienenden Hexoxyds in diese Verbindung übergeführt.

Bei Fraktion III handelt es sich um eine farblose, schwerflüchtige, ölige Flüssigkeit, die wir auf Grund dieses Verhaltens für Chlorheptoxyd hielten, eine Annahme, die sich als richtig erwies.

Die Identifizierung führ. ten wir zunächst auf $\mathrm{ch}$ e mi . schem Wege durch. In der wässerigen Lösung der Sub$\operatorname{stanz}$ wurden weder Fluor, noch Chlor, Hy pochlorit, Chlorit- oder Chlorat-Ionen vorgefunden. Als einzige chlorhaltige Säure blieb also nur die Überchlorsäure übrig. Der Gesamtgehalt an Säure in der Lösung entsprach dem, den man bei Vorliegen von Heptoxyd erwarten sollte.

Es wurden ferner die Dampfdrucke verschiedener Proben gemessen. Wie Abb. 3 zeigt, stimmen die Meßwerte in befriedigender Weise mit den von GoODEve ${ }^{25}$ ) bestimmten Werten des reinen Chlorhept-

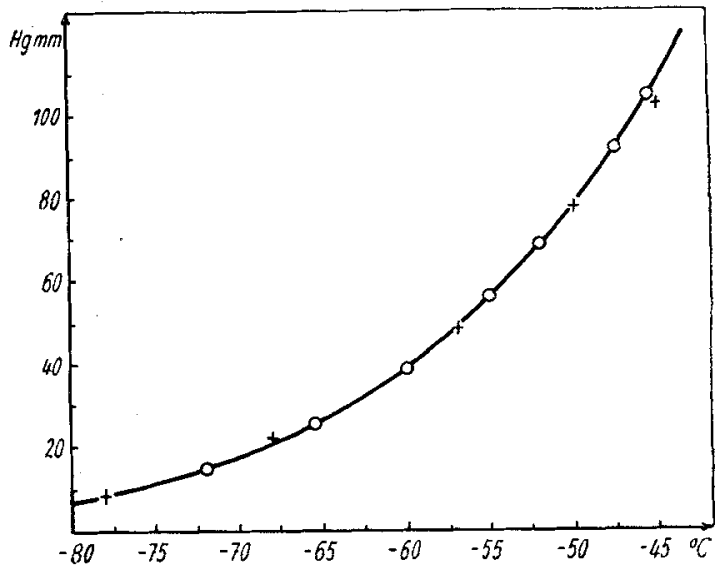

Abb. 2. Dampfdruckkurve des $\mathrm{FClO}_{2}$. $\bigcirc$ Schmiтz u. SchumaChER, + Versuch 19

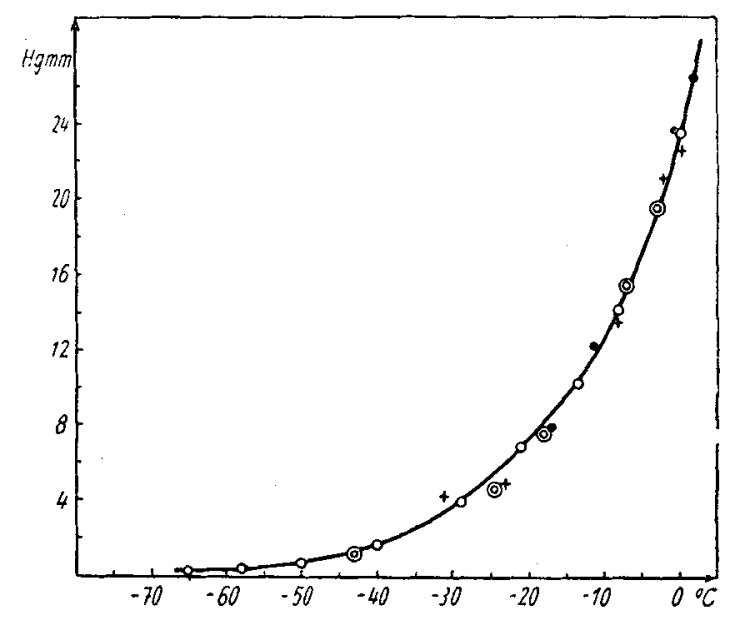

Abb. 3. Dampfdruckkurve des $\mathrm{Cl}_{2} \mathrm{O}_{7}$. O G. u. P., - Versuch 13, + Versuch 14, 0 Versuch 16 oxyds überein.

Schließlich wurde noch eine bestimmte Menge dieser Fraktion durch langsames Erwärmen zersetzt und der Enddruck nach völligem Zerfall bestimmt. Die Druckzunahme

${ }^{25}$ ) C. F. Goodeve u. J. Powney, J. chem. Soc. [London] 1932, 2078.

Z, anorg. allg. Chemie. Bd. 286. 
betrug etwa $350 \%$, wie man es bei einer Verbindung der Formel $\mathrm{Cl}_{2} \mathrm{O}_{7}$ erwarten sollte. Die Bestimmung des Chlorgehaltes der Zersetzungsprodukte durch Titration ergab ebenfalls Werte, die zu dieser Formel paßten.

Es kann hiernach als sicher angesehen werden, daß Fraktion III aus reinem Chlorheptoxyd besteht. Der Menge nach stellt sie etwa $20-30 \%$ des umgesetzten Hexoxydes dar.

Die erste Fraktion ist mengenmäßig die bei weitem kleinste. Es handelt sich je nach den Versuchsbedingungen um etwa $10-20 \mathrm{~mm}$ $\mathrm{Hg}$ bei $15^{\circ} \mathrm{C}$. Wie die Titrationen ergaben, besteht sie zum großen Teil aus freiem Chlor. Es wurden jedoch in der wässerigen Lösung auch in geringer Menge Fluor-, Chlorit- und Chlorat-Ionen gefunden. Die Werte, bedingt durch die geringen zur Verfügung stehenden Mengen, streuten jedoch so sehr, daß keine sicheren Schlüsse auf eine bestimmte Verbindung gezogen werden konnten.

In jedem Fall muB es sich um eine leichtflüchtige Substanz handeln. Von den bekannten Fluor, Chlor und Sauerstoff enthaltenden Verbindungen genügen das Perchlorylfluorid $\left(\mathrm{FClO}_{3}\right)$, sein isomeres, $\mathrm{ClO}_{2} \mathrm{OF}$, dessen Existenz allerdings noch nicht ganz gesichert erscheint, und das Fluorperchlorat $\left(\mathrm{FClO}_{4}\right)^{26}$ ) dieser Bedingung. Da das Perehlorylfluorid nur sehr schwer hydrolysieren soll, kann es ausgeschlossen werden, so daß lediglich Fluorperchlorat $\mathrm{FCIO}_{4}$ übrig bleibt. Es läßt sich jedoch, wie bereits ausgeführt wurde, nicht mit Sicherheit entscheiden, ob es sich um diese oder eine andere noch unbelsannte Substanz handelt. Gewisse Gründe, wie z. B. die Tatsache, daß Fraktion II, falls sie nicht vollständig von den leichter flüchtigen Teilen getrennt war, bisweilen beim Einlassen der Lösungsflüssigkeit schwache Explosionserscheinungen zeigte, sprechen allerdings für das instabile und leicht explosiv zerfallende $\mathrm{FClO}_{4}$.

Es wurde noch eine Reihe von Versuchen verschiedener Art angestellt mit dem Ziel, Näheres über die Natur der auftretenden Reaktionen. zu erfahren.

1. Zunächst wurde der Fluordruck variiert. Während die Mehrzahl der Versuche mit einem Fluor Anfangsdruck von etwa $250 \mathrm{~mm} \mathrm{Hg}$ begonnen und dementsprechend mit einem Druck von etwa $150 \mathrm{~mm} \mathrm{Hg}$ aufhörten, so daß man mit einer mittleren Konzentration von etwa $200 \mathrm{~mm} \mathrm{Hg}$ rechnen kann, wurde nunmehr eine Serie von Versuchen mit einer mittleren Konzentration von $40 \mathrm{~mm} \mathrm{Hg}$ durchgeführt. Bei diesen Versuchen wurde mit etwa $50 \mathrm{~mm} \mathrm{Hg}$ Fluor begonnen. Nachdem ungefähr dreiviertel hiervon verbraucht waren, was man aus der Druckerhöhung leicht feststellen kann, wurde das Reaktionsgefä $B$ auf $0^{\circ} \mathrm{C}$ abgekühlt, abermals $50 \mathrm{~mm} \mathrm{Hg}$ Fluor zugegeben, und die Reaktion bei der ursprünglichen Temperatur weiterverfolgt. Nach Verbrauch der entsprechenden Menge Fluor wurde dieses Verfahren bis zum Reaktionsende wiederholt.

Diese Versuche hatten das überraschende Ergebnis, daß die Reaktionszeit, das ist die Zeit, die verstreicht bis das gesamte Chlorhexoxyd verschwunden ist, bei einem Fluorpartialdruck von $\sim 40 \mathrm{~mm}$ die gleiche

26) G. H. Rohrback u. G. H. Cady, J. Amer. chem. Soc. 69, 677 (1947). 
ist, wie bei einem Druck von $\sim 200 \mathrm{~mm}, \mathrm{~d}$. h. daß sie unabhängig vom Fluordruck ist. Die Art der Reaktionsprodukte bleibt ebenfalls unverändert. Die Menge des gebildeten Chlorheptoxyds bleibt, soweit feststellbar, die gleiche, während die des Chlorylfluorids in geringem Maße abnimmt. Die Mengen von freiem Chlor und besonders freiem Sauerstoff erhöhen sich jedoch in beträchtlichem Maße. Da die Menge des freien Chlors an sich gering ist, so rufen bereits absolut geringe Mengen, prozentual große Änderungen hervor. Ferner ist der Druck der nicht kondensierbaren Gase nach der Reaktion erheblich größer als der Anfangsdruck des Fluors, es ist also ein Überschuß an freiem Sauerstoff vorhanden; die Gesamtdruckerhöhung übertrifft in stärkerem Maße die Summe der kondensierbaren Produkte als bei den Versuchen mit hohem Fluordruck.

Diese Resultate werden noch deutlicher erkennbar, wenn man zum Vergleich einen Versuch mit extrem hohem Fluordruck $(400 \mathrm{~mm}$ $\mathrm{Hg}$ ) heranzieht. In diesem Fall ist der Uberschuß an freiem Sauerstoff besonders klein, während die Menge der nicht mit Sicherheit identifizierten Verbindung relativ groß ist.

Es hat hiernach den Anschein, als ob das Fluor mit dem Chlorhexoxyd und seinem monomeren $\mathrm{ClO}_{3}$ keine direkte Reaktion eingeht, da $\beta$ es aber mit den Zerfallsprodukten dieses Oxydes reagiert und verhindert, daß diese sich weiter zu Chlor und Sauerstoff umsetzen.

Falls diese Annahme richtig ist, dann sollte die Zeit, innerhalb der eine bestimmte Menge von Chlorhexoxyd verschwindet, $d . h$. zerfällt, von der Anwesenheit des Fluors unabhängig sein. Wir untersuchten daher die Geschwindigkeit des Hexoxydzerfalls bei Abwesenheit von Fluor.

Nun kann die Verdampfungsgeschwindigkeit einer Flüssigkeit und die Höhe ihres stationären Dampfdruckes dadurch beeinflußt werden, daß sich Reaktionsprodukte in der Flüssigkeit lösen. Auch ist es denkbar, daß die Zerfallsprodukte Reaktionen eingehen, die das Ausgangsprodukt rückbilden. Um diese Einflüsse auszuschließen gingen wir bei den Versuchen bei Abwesenheit von Fluor so vor, daß wir die Reaktion alle zehn Minuten unterbrachen, das Reaktionsgefäß auf $-10^{\circ} \mathrm{C}$ abkühlten, die entwickelten Gase abpumpten, dann wieder auf Reaktionstemperatur aufwärmten und so fortfuhren, bis alles Hexoxyd verschwunden war.

Es zeigte sich, daß innerhalb der Versuchsgenauigkeit kein Unter schied in den Zerfallszeiten des Chlorhexoxyds bei den Versuchen mit und ohne Fluor festzustellen war.

Bei den Versuchen mit Fluor haben wir die Reaktionszeiten zahlreicher Versuche bei 22, 25, 40 und $48^{\circ} \mathrm{C}$ gemessen. Es war erstaunlich, 
wie gut reproduzierbar diese Zeiten, natürlich jeweils auf die gleiche Hexoxydmenge bezogen, waren, obwohl bei diesen Versuchen die Oberflächenverhältnisse oftmals recht verschieden waren, da je nach den Herstellungsbedingungen das Chlorhexoxyd manchmal in Form vieler kleiner und in anderen Fällen in Form weniger großer Tropfen vorhanden war, und obwohl weiterhin zu berücksichtigen ist, daß sich während der Reaktion gebildete Produkte kondensierten und lösten. Nach allem diesem sollte man aber stark streuende Ergebnisse erwarten, falls die Verdampfungsgeschwindigkeit irgendwie für die späteren Ereignisse von Bedeutung ist.

Die beobachtete Reproduzierbarkeit der Versuchszeiten besagt also, daß die Verdampfungsgeschwindigkeit keine Rolle bei den späteren Reaktionen spielt, bzw. daß die Verdampfungsgeschwindigkeit stets groß genug ist, um den der Temperatur entsprechenden Partialdruck an Dampf aufrechtzuerhalten.

Die Geschwindigkeit, mit der das Hexoxyd verschwindet, wird also durch die Geschwindigkeit der Zerfallsreaktionen des Trioxyds bestimmt. Da diese durch die Größe und Art der jeweiligen Oberfläche nicht beeinflußt werden, kann angenommen werden, daß es sich um homogene Gasreaktionen handelt.

2. Weitere Versuche betrafen das Chlorheptoxyd. Zunächst war festzustellen, ob die nach Reaktionsende gefundene Menge der während der Reaktion gebildeten entspricht, oder ob nicht bereits Anteile durch thermischen Zerfall oder Reaktion mit dem Fluor verbraucht worden waren.

a) Etwa $30 \mathrm{~mm} \mathrm{Hg}$ dieses Oxydes - sie wurden der Fraktion III entnommen wurden bei Gegenwart von etwa $200 \mathrm{~mm} \mathrm{Hg} \mathrm{Fluor} \mathrm{eine} \mathrm{Stunde} \mathrm{bei} 40^{\circ} \mathrm{C}$ gehalten. Es zeigte sich, daß während dieser Zeit keinerlei Reaktion eingetreten war.

b) Wir erhitzten etwa $35 \mathrm{~mm} \mathrm{Hg}$ reines Chlorheptoxyd frei von allen Beimengungen auf $40^{\circ} \mathrm{C}$ und beobachteten den Druck über mehrere Stunden. Es zeigte sich, daß das Heptoxyd bei $40^{\circ} \mathrm{C}$ noch als thermisch völlig stabil anzusehen ist. Erst beim Erhitzen auf $80^{\circ} \mathrm{C}$ konnte ein Zerfall festgestellt werden; bei $100^{\circ} \mathrm{C}$ betrug der Druckanstieg etwa $1 \mathrm{~mm} \mathrm{Hg} /$ Minute.

Aus den Versuchen a) und b) folgt, daß das Chlorheptoxyd unter unseren Versuchsbedingungen während der Reaktion weder zerfällt noch durch Reaktion mit Fluor verbraucht wird.

Es war nunmehr festzustellen, ob tür die Bildung der von uns gefundenen relativ großen Mengen an Chlorheptoxyd die Anwesenheit von Fluor notwendig ist oder nicht. Es war zwar bereits von Goodeve und RICHARDSON ${ }^{14}$ ) festgestellt worden, daß sich unter den beim thermischen Zerfall des Chlorhexoxyds auftretenden Produkten auch das Heptoxyd 
befindet, doch fehlen alle Angaben über die auftretenden Mengen und seinen Bildungsmechanismus.

c) Wir haben Chlorhexoxydmengen von der Größenordnung, wie wir sie bei unseren Versuchen benutzten, ohne jeden Fremdgaszusatz sowohl bei 25 wie bei $40^{\circ} \mathrm{C}$ thermisch zerfallen lassen und die Zerfallsprodukte auf etwa vorhandenes Heptoxyd untersucht. Wir kühlten zu diesem Zwecke die gebildeten Gase auf etwa $-60^{\circ} \mathrm{C}$ ab und evakuierten dann bei dieser Temperatur. Zurückblieb eine farblose Flüssigkeit, die sich als Chlorheptoxyd erwies, und die ihrer Menge nach durchaus den bei den Versuchen mit Fluor auftretenden Chlorheptoxydmengen entsprach.

Es steht somit fest, daß das Chlorheptoxyd zu seiner Bildung nicht der Anwesenheit des Fluors bedarf, sondern daß es bereits beim thermischen Zerfall des Hexoxydes gebildet wird.

Eine Übersicht über unsere Versuchsdaten gibt Tab. 1. In der Tab. 1 sind die Versuchsdaten zusammengefaßt.

In dieser bedeuten: $\mathrm{N}_{\mathrm{v}}$ die Versuchsnummer, $\mathrm{T}$ die Temperatur in ${ }^{\circ} \mathrm{C}$, $t$ die Zeit in Minuten, $\mathrm{Cl}_{2} \mathrm{O}_{6}$ den der Hexoxydmenge entsprechenden Gasdruck, unter der Annahme berechnet, daB es als Dimeres vorliegt, $p_{a}$ den Anfangsdruck des Fluors und seiner inerten Verunreinigungen, $F_{2}$ den des reinen Fluors, $F_{2 m}$ den mittleren Fluordruck während des Versuches, $\Sigma \Delta \mathrm{p}$ die Gesamtdruckzunahme während der Reaktion, $\mathrm{p}_{\mathrm{n} \mathbf{k}}$ den Druck der bei $-183^{\circ} \mathrm{C}$ nicht kondensierbaren Gase, $\mathrm{p}_{\mathrm{kR}}$ den der bei $-183^{\circ}$ kondensierbaren Reaktionsprodukte, $\Delta_{1}$, die Differenz zwischen $p_{n k}$ und $p_{a}$, ist ein Maß für den Überschuß an freiem Sauerstoff. $\Delta_{1}$ kann wegen des zur $\mathrm{Cl}_{2} \mathrm{O}_{7}$-Bildung benötigten Sauerstoffverbrauches negative Werte annehmen. $\Delta_{2}$ ist die Differenz zwischen $\Sigma \Delta \mathrm{p}$ und $\mathrm{p}_{\mathrm{k} R}, \mathrm{FClO}_{2}$, $\mathrm{Cl}_{2} \mathrm{O}_{7}, \mathrm{FClO}_{2} / \mathrm{Cl}_{2} \mathrm{O}_{7}, \mathrm{Cl}_{2}$ und $\mathrm{X}$ stellen die Drucke bzw. den Quotienten der entsprechenden Substanzen dar.

Alle Drucke sind in $\mathrm{mm} \mathrm{Hg}$ von $15^{\circ} \mathrm{C}$ angegeben. $\mathrm{Zu}$ bemerken ist, da B alle Daten experimentelle Werte darstellen, bis auf die Einzelwerte für $\mathrm{Cl}_{2}$ und $\mathrm{X}$, deren Summe experimentell bestimmt wurde. Der $\mathrm{Cl}_{2}$-Wert wurde unter der Annahme berechnet, da B X die Formel $\mathrm{FClO}_{4}$ zukommt. Da der Hauptteil des freien Chlors durch den Überschuß an freiem Sauerstoff und die Menge an $\mathrm{Cl}_{2} \mathrm{O}_{7}$ bestimmt wird, wird der Chlor-Wert durch obiges Ermittelungsverfahren in der Regel nur wenig verändert. Die durch Titration der Fraktion I erhaltenen Chlor-Werte weichen von den angegebenen ,theoretischen" Werten nur wenig ab.

\section{Diskussion der Ergebnisse}

Aus den beschriebenen Versuchen ergibt sich das folgende Bild: Chlorhexoxyd $\mathrm{Cl}_{2} \mathrm{O}_{6}$ und das mit ihm im Gleichgewicht stehende Chlortrioxyd $\mathrm{ClO}_{3}$ reagieren nicht oder zum mindesten nur mit einer äußerst geringen Geschwindigkeit mit Fluor. Das Trioxyd geht seinerseits verschiedene Zerfallsreaktionen ein, die sich hinsichtlich ihrer Geschwindigkeit und, obwohl natürlich die Endprodukte des Zerfalls Chlor und Sauerstoff sind, auch der Art der gebildeten Zwischenprodukte nach in charakteristischer Weise voneinander unterscheiden. 
Tabelle 1

Zusammenstellung der

\begin{tabular}{|c|c|c|c|c|c|c|c|c|}
\hline $\mathrm{N}_{\mathrm{v}}$ & $\begin{array}{l}\mathrm{T} \\
{ }^{\circ} \mathrm{C}\end{array}$ & $\begin{array}{c}\mathrm{Cl}_{2} \mathrm{O}_{6} \\
\mathrm{~mm} \mathrm{Hg}\end{array}$ & $\stackrel{t}{\mathbf{t}}$ & $\underset{\mathrm{mm}}{\mathrm{p}_{\mathrm{a}}} \mathrm{Hg}$ & $\begin{array}{c}\mathrm{F}_{2} \\
\mathrm{~mm} \mathrm{Hg}\end{array}$ & $\begin{array}{c}\mathrm{F}_{2} \mathrm{~m} \\
\mathrm{~mm} \mathrm{Hg}\end{array}$ & $\begin{array}{l}\Sigma \Delta \mathrm{p} \\
\mathrm{mm} \mathrm{Hg}\end{array}$ & $\begin{array}{c}p_{\mathbf{n k}} \\
\mathrm{mm} \mathrm{Hg}\end{array}$ \\
\hline 10 & 22 & 147,1 & 1200 & 271,6 & 251,4 & 202,6 & 242,4 & 270,0 \\
\hline 15 & 25 & 119,1 & 810 & 246,4 & 232,2 & 193,8 & 200,0 & 247,2 \\
\hline 21 & 25 & 114,4 & 790 & 273,3 & 264,8 & 225,5 & 199,7 & 278,0 \\
\hline 23 & 25 & 103,5 & 690 & 257,9 & 248,4 & 215,0 & 167,4 & 252,8 \\
\hline 8 & 40 & 147,5 & 133 & 273,9 & 251,8 & 201,8 & 281,8 & 290,6 \\
\hline 11 & 40 & 150,8 & 136 & 268,1 & 250,5 & 198,0 & 290,5 & 284,5 \\
\hline 13 & 40 & 146,7 & 132 & 275,3 & 256,0 & 206,2 & 261,9 & 285,1 \\
\hline 14 & 40 & 109,2 & 99 & 271,1 & 254,2 & 217,4 & 192,2 & 274,7 \\
\hline 16 & 40 & 159,7 & 144 & 285,3 & 269,2 & 215,3 & 296,7 & 314,4 \\
\hline 17 & 40 & 129,3 & 117 & 272,7 & 264,0 & 222,1 & 225,3 & 282,9 \\
\hline 18 & 40 & 164,8 & 149 & 255,8 & 248,4 & 193,4 & 311,0 & 285,1 \\
\hline 27 & 40 & 160,6 & $145^{\bullet}$ & 277,2 & 266,4 & 214,6 & 277,6 & 288,7 \\
\hline 30 & 40 & 98,8 & 89 & 277,3 & 266,8 & 233,5 & 170,2 & 281,2 \\
\hline 34 & 48 & 148,1 & 47 & 263,9 & 252,5 & 205,1 & 260,2 & 283,5 \\
\hline $\left.20^{27}\right)$ & 40 & 137,5 & 174 & 150,7 & & 60 & 268,1 & 191,6 \\
\hline $\left.22^{27}\right)$ & 40 & 140,4 & 178 & 185,0 & & 40 & 265,8 & 227,9 \\
\hline 31 & 40 & 166,2 & 150 & 439,0 & 424,1 & 368,3 & 283,1 & 441,4 \\
\hline
\end{tabular}

Von Bodenstein, Schumacher und Mitarbeitern ${ }^{y}$ ) war bereits angenommen worden, daß das Hexoxyd in der Gasphase nahezu völlig in 2 Molekeln Trioxyd dissoziiert ist. Von Goodeve und Mitarbeitern ${ }^{28}$ ) wurde der exakte Beweis hierfür geliefert und auch die Dissoziationswärme ${ }^{29}$ ) bestimmt. Sie beträgt für die Dissoziation im flüssigen Zustand $1700 \pm 500 \mathrm{cal}$.

Úber die Art des thermischen Zerfalls des Trioxyds hat man sich seit langem Gedanken gemacht, auch wurden von verschiedenen Seiten experimentelle Untersuchungen durchgeführt, mit dem Ziel, seinen Zerfallsmechanismus aufzuklären. Endgültige Klarheit konnte jedoch bisher nech nicht erreicht werden.

Von SchuMaCheR $\left.{ }^{9}\right)^{10}$ ) war bei Untersuchungen des durch Chlor katalysierten Ozonzerfalls, bei dem sich $\mathrm{ClO}_{3}$ als Zwischenprodukt bildet, das Auftreten der bimolekularen Reaktion:

$$
\mathrm{ClO}_{3}+\mathrm{ClO}_{3}=\mathrm{Cl}_{2}+3 \mathrm{O}_{2}
$$

27) In den Versuchen 20 und 22 wurde das Fluor schubweise zugegeben.

${ }^{28}$ ) C. F. Gooneve u. S. Tond, Nature 132, 514 (1933).

${ }^{29}$ ) J. Farquharson, C. F. Goodeve u. F. D. Richardson, Trans. Faraday Soc. 32, 790 (1936). 
Tabelle 1

Versuchsergebnisse

\begin{tabular}{|c|c|c|c|c|c|c|c|}
\hline $\begin{array}{c}\mathrm{p}_{\mathrm{kR}} \\
\mathrm{mm} \mathrm{Hg}\end{array}$ & $\Delta_{1}$ & $\Delta_{2}$ & $\begin{array}{c}\mathrm{FClO}_{2} \\
\mathrm{~mm} \mathrm{Hg}\end{array}$ & $\begin{array}{l}\mathrm{Cl}_{2} \mathrm{O}_{7} \\
\mathrm{~mm} \mathrm{Hg}\end{array}$ & $\begin{array}{l}\mathrm{FClO}_{2} \\
\mathrm{Cl}_{2} \mathrm{O}_{7}\end{array}$ & $\begin{array}{c}\mathrm{Cl}_{2} \\
\mathrm{~mm} \mathrm{Hg}\end{array}$ & $\begin{array}{c}\mathrm{X} \\
\mathrm{mm} \mathrm{Hg}\end{array}$ \\
\hline 244,2 & $-1,6$ & $-1,8$ & 195,0 & 35,0 & 5,6 & 9,1 & 11,0 \\
\hline 198,7 & $-0,8$ & 1,3 & 153,5 & 31,2 & 4,9 & 8,1 & 5,9 \\
\hline 195,6 & $-4,7$ & 4,1 & 157,0 & 25,3 & 6,2 & 7,9 & 5,4 \\
\hline 172,8 & 5,1 & $-5,4$ & 133,5 & 25,0 & 5,3 & 6,2 & 11,1 \\
\hline 265,0 & 16,7 & 16,8 & 200 & 35 & 5,7 & 11,7 & 1,6 \\
\hline 273,7 & 16,4 & 16,8 & 210 & 38 & 5,6 & 7,8 & - \\
\hline 251,7 & 9,8 & 10,2 & 198,9 & 35,0 & 5,7 & 10,1 & 4,1 \\
\hline 189,1 & 3,6 & 3,1 & 147,2 & 27,6 & 5,3 & 6,7 & 2,6 \\
\hline 267,8 & 29,1 & 28,9 & 215,5 & 34,7 & 6,2 & 16,8 & 0,8 \\
\hline 215,3 & 10,2 & 10,0 & 167,3 & 34,3 & 4,9 & 10,0 & 8,7 \\
\hline 281,2 & 29,3 & 29,8 & 220 & 40 & 5,4 & 14,8 & - \\
\hline 266,6 & 11,5 & 11,0 & 207,4 & 40,8 & 4,8 & 12,9 & 6,3 \\
\hline 166,3 & 3,9 & 3,9 & 133,2 & 25,0 & 5,3 & 6,2 & 1,9 \\
\hline $241, \overline{5}$ & 19,6 & 18,7 & 182,8 & 40,7 & 4,5 & 14,2 & 3,6 \\
\hline 226,6 & 40,9 & 41,5 & 178,3 & 33,2 & 5,4 & 15,1 & - \\
\hline 232,9 & 42,9 & 32,9 & 185,1 & 30,1 & 6,1 & 17,7 & - \\
\hline 287,8 & 2,4 & 4,3 & 215,1 & 42,4 & 5,1 & 11,2 & 10,1 \\
\hline
\end{tabular}

sichergestellt und ihre Geschwindigkeitskonstante und Aktivierungsenergie $\left(q_{1}\right)$ quantitativ bestimmt worden:

$$
\mathrm{q}_{1} \simeq 11,5 \mathrm{kcal}
$$

Von dem gleichen Autor ${ }^{18}$ ) war festgestellt worden, daß sich beim thermischen Zerfall des Hexoxydes in erheblichem Ausmaße Chlordioxyd bildet, dessen Identifizierung auf spektroskopischem Wege durchgeführt worden war.

Von Rollefson ${ }^{30}$ ) und insbesondere auch von Goodeve ${ }^{14}$ ) und Mitarbeitern ist das Chlorhexoxyd eingehend untersucht worden.

RollefsoN und Mitarbeiter bestimmten bei verschiedenen Temperaturen zwischen $0^{\circ}$ und $30^{\circ} \mathrm{C}$ die Menge der kondensierbaren und der nichtkondensierbaren $\left(\mathrm{O}_{2}\right)$ Reaktionsprodukte. Hierbei zeigte sich, daß sich bei tiefen Temperaturen zu einem hohen Prczentsatz Chlordioxyd bildete und $d a ß$ der relative Gehalt der Zerfallsprodukte an diesem Oxyd mit steigender Temperatur abnahm. Aus der Temperaturabhängigkeit der Geschwindigkeit der Druckzunahme berechneten sie für die Aktivierungswärme des Zerfalls von flüssigem Chlorhexoxyd Werte, die zwisehen 20,8 und 26,2 kcal liegen.

Von Goodeve und Richardson wurden ähnliche Versuche, jedoch mit größerer Genauigkeit durchgeführt. Sie finden für den Temperaturkoeffizienten des Zerfalls von flüssigen Chlorhexoxyds aus zwischen $5^{\circ}$ und $15^{\circ} \mathrm{C}$ durchgeführten Versuchen einen Wert von 3,8 entsprechend einer Aktivierungsenergie von $22 \pm 2 \mathrm{kcal}$.

so) A. C. Byrns u. G. K. Rollefson, J. Amer. chem. Soc. 56, 2245 (1934). 
Nun kann man gegen die Berechnung einer Aktivierungsenergie unter den vorliegenden Bedingungen erhebliche Einwände erheben. Die Autoren sèlbst, Goodeve und RrChardson, weisen darauf hin, daB der so bestimmten Aktivierungsenergie nur der Charakter einer ,scheinbaren" Aktivierungsenergie zukommt. Da nämlich das Chlorhexoxyd verschiedene Zerfallsreaktionen.eingeht, so ist die gemessene Druckzunahme durch das Zusammenspiel verschiedener voneinander unabhängiger Reaktionen bedingt. Der aus dem Temperaturkoeffizienten der Druckzunahme berechnete Wert kann also nicht mehr ohne weiteres als Aktivierungsenergie einer bestimmten Reaktion angesehen werden. Hierzu kommt noch, das das thermisch wenig stabile Chlordioxyd zum mindesten bei höheren Temperaturen (z. B. bei den Versuchen von Byrns und Rollsfson) durch seinen Eigenzerfall die Resultate fälschen kann.

Goodeve und Pichardson haben ferner die Verdampfungs- und die Sublimationswärme des Hexoxydes bestimmt, und geben folgende Werte an: $\mathrm{L}_{\mathrm{v}}=9,5 \pm 1 \mathrm{kcal}$; $I_{\mathrm{s}}=12,3 \pm 0,5 \mathrm{kcal}$. Sie teilen ferner mit, daß bei Temperaturen oberhalb von $-20^{\circ} \mathrm{C}$ als Zerfallsprodukte Chlor, Chlordioxyd und Chlorheptoxyd auftreten. Es fehlen jedoch alle Angaben über die Mengenverhältnisse, in denen diese Produkte erscheinen, wie über den Mechanismus der Reaktionen, die zu ihrer Bildung führen.

Die von uns durchgeführten Versuche gestatten es, den Zerfall des Chlorhexoxydes weitgehend zu klären und die verschiedenen Reaktionen quantitativ zu beschreiben. Da nämlich das Fluor die Eigenschaft hat, das thermisch instabile Chlordioxyd in schneller Reaktion in das relativ stabile Chlorylfluorid überzuführen, gelingt es, übersichtliche Verhält. nisse zu schaffen.

Zunächst zeigen unsere Versuche, daß sich die Reaktionszeiten bei $25^{\circ}$ und $40^{\circ} \mathrm{C}$ um etwa den Faktor 7,5 voneinander unterscheiden. Dies entspricht - in bester Ubereinstimmung mit dem von Goodeve und RichaRdson auf gänzlich anderem Wege erhaltenen Werte - einem Temperaturkoeffizienten von 3,84 .

Ferner stellten wir fest, daß bei Anwesenheit von genügend Fluor $70-75 \%$ des Hexoxydes in Chlorylfluorid und etwa $25 \%$ in Chlorheptoxyd umgewandelt werden und daß sich dieses Verhältnis zwischen $22^{\circ}$ und $48^{\circ} \mathrm{C}$ nur in sehr geringem Maße ändert.

Wir wissen also nunmehr, daß etwa $70 \%$ des Hexoxydes in Chlordioxyd umgesetzt werden. Dies kann nur nach Gl. (2) geschehen ${ }^{31}$ ):

$$
\mathrm{ClO}_{3}+\mathrm{ClO}_{3}=2 \mathrm{ClO}_{2}+\mathrm{O}_{2} \text {. }
$$

Die Aktivierungsenergie von Reaktion (2) läßt sich recht genau festlegen. Wir haben hierfür zwei Wege.

Zunächst wissen wir aus dem Vergleich der Mengen von freiem Chlor und von Chlorylfluorid der bei der tieferen Temperatur durchge-

s1) Eine Dissoziation des $\mathrm{ClO}_{3}$ nach $\mathrm{ClO}_{3}=\mathrm{ClO}_{2}+\mathrm{O}$ scheidet aus energetischen Gründen aus. 
führten Versuche - hier kann die Chlorbildung durch Zerfall des Chlordioxyds vernachlässigt werden, - daß Reaktion (2) mindestens $20 \mathrm{mal}$ so schnell verläuft wie Reaktion (1). Bei Annahme gleicher sterischer Faktoren für (1) und (2) würde dies einem Unterschied in den Aktivierungswärmen von $1,5-2 \mathrm{kcal}$ entsprechen. Nun dürfte aber (2) einen größeren sterischen Faktor haben als (1), so daß es als durchaus möglich angesehen werden kann, daß die Geschwindigkeitsunterschiede beider Reaktionen durch die Verschiedenheit ihrer sterischen Faktoren bedingt sind. Auf jeden Fall kann man annehmen, daß beide Reaktionen Aktivierungsenergien der gleichen Größenordnung besitzen:

$$
q_{2} \simeq q_{1} \text {. }
$$

Der zweite Wèg zur Bestimmung von $q_{2}$ geht auf die Messung des Temperaturkoeffizienten der Gesamtzerfallsgeschwindigkeit zurück. Da wir nunmehr wissen, daß das Chlordioxyd das Hauptreaktionsprodukt ist und aus der Konstanz der Mengenverhältnisse von Heptoxyd zu Chlorylfluorid schließen können, daß der Temperaturkoeffizient der Chlorheptoxydbildung nicht wesentlich von der der Chlordioxydbildung abweicht, so dürfte die aus dem Temperaturkoeffizienten der Gesamtreaktion berechnete sogenannte ,scheinbare“ Aktivierungsenergie in 1. Annäherung der Reaktion (2) zugeschrieben werden können. Da der gemessene Temperaturkoeffizient sich auf das Verschwinden des flüssigen Hexoxydes bezieht, muß zur Berechnung der Aktivierungsenergie der Gasreaktion von dem so berechneten Wert noch die Verdampfungswärme des Hexoxydes und seine Dissoziationsenergie abgezogen werden.

$$
\mathrm{q}_{2}=22-9,5-1,5=11 \pm 2 \mathrm{kcal}
$$

Wir gelangen also auf beiden Wegen zu sehr ähnlichen Werten. Man kann also annehmen, daß $q_{2}=\mathbf{1 1 , 5} \pm 2$ keal ist.

Die Bildung des Chlorheptoxydes erfolgt nach unseren Versuchen ohne jede Einwirkung des Fluors durch Reaktion von Zerfallsprodukten des Hexoxydes. Es ist naheliegend, anzunehmen, daß es durch Anlagerung zweier Radikale (des $\mathrm{ClO}_{4}$ und des $\mathrm{ClO}_{3}$ ) entsteht. Für die Bildung des $\mathrm{ClO}_{4}$ schlagen wir eine der folgenden Reaktionen vor:

oder

anschließend:

$$
\mathrm{ClO}_{3}+\mathrm{ClO}_{3}=\mathrm{ClO}_{4}+\mathrm{ClO}_{2}
$$

$$
\mathrm{Cl}_{2} \mathrm{O}_{6 \text { gast. }}=\mathrm{ClO}_{4}+\mathrm{ClO}_{2} \text {, }
$$

$$
\mathrm{ClO}_{3}+\mathrm{ClO}_{4}=\mathrm{Cl}_{2} \mathrm{O}_{7}
$$


Ob die $\mathrm{ClO}_{4}$-Bildung nach (3a) oder nach (3b) erfolgt, läßt sich nicht entscheiden. Beide Wege erscheinen möglich. Da $\mathrm{Cl}_{2} \mathrm{O}_{6(\text { gass. })}$ im Gleichgewicht mit $\mathrm{ClO}_{3}$ steht, so wird seine Konzentration durch das Quadrat der $\mathrm{ClO}_{3}$-Konzentration bestimmt, so daß auch kinetisch kein Unterschied vorhanden ist. Die Aktivierungsenergie von (3a) bzw. (3 b) dürfte nicht stark von der der Reaktionen (2) und (1) abweichen. Wie bereits erwähnt, sind die Mengen an Chlorylfluorid und Chlorheptoxyd von der gleichen Größenordnung und ihr Verhältnis ist bei 22 und $48^{\circ} \mathrm{C}$ innerhalb der Versuchsgenauigkeit das gleiche:

$$
\mathrm{q}_{1} \simeq \mathrm{q}_{2} \simeq \mathrm{q}_{3} .
$$

An Reaktion (2) schließt sich bei Gegenwart von Fluor unmittelbar Reaktion (5) an:

$$
\mathrm{ClO}_{2}+\mathrm{F}_{2}=\mathrm{FClO}_{2}+\mathrm{F} \text {. }
$$

Das hierbei gebildete Fluoratom kann entweder mit einer zweiten Chlordioxydmolekel reagieren, zu Fluormolekeln im Gasraum oder an der Wand rekombinieren oder möglicherweise zur Bildung des leichtflüchtigen, von uns nicht näher identifizierten Produktes führen. Falls es sich hierbei um das $\mathrm{FClO}_{4}$ handeln sollte, so ist dessen Bildung durch Reaktion $(5 c)$ gegeben.

$$
\begin{aligned}
\mathrm{ClO}_{2}+\mathrm{F} & \rightarrow \mathrm{FClO} \\
\mathrm{F}+\mathrm{F} & \rightarrow \mathrm{F}_{2}, \\
\mathrm{~F}+\mathrm{ClO}_{4} & \left.\rightarrow \mathrm{FClO}_{4}{ }^{32}\right) .
\end{aligned}
$$

Reaktion (5) ist von Schumacher und Mitarbeitern $\left.{ }^{7}\right)^{8}$ ) eingehend untersucht worden. Sie hat eine Aktivierungsenergie von etwa $8 \mathrm{kcal}$ und einen sterischen Faktor $\alpha$ von etwa $10^{-4}$. Es handelt sich also um eine mit großer Geschwindigkeit verlaufenden Reaktion, die aber doch wieder nicht so schnell ist, um momentan alles Chlordioxyd aus dem Reaktionssystem zu entfernen. Man kann leicht ausrechnen, daß unter unseren Versuchsbedingungen, eine Chlordioxydmolekel im Mittel $1 / 2$ bis 1 Minute existiert, bis sie in Chlorylfluorid übergeführt ist.

Nun ist bekanntlich das Chlordioxyd selbst eine sehr instabile Verbindung, deren thermischer Zerfall von Schumacher und Stieger ${ }^{33}$ ) gründlich untersucht worden ist. Es zerfällt nach diesen Autoren in

32) Das $\mathrm{FClO}_{4}$ könnte sich natürlich auch durch direkte Reaktion zwischen $\mathrm{ClO}_{4}$ und einer Fluormolekel bilden. $\mathrm{D}_{\mathrm{O}-\mathrm{F}}>\mathrm{D}_{\mathbf{F}_{2}}$.

33) H. J. Schunacher u. G. Stieger, Z. physik. Chem., Abt. B 7, 363 (1930). 
einer komplizierten, heterogenen Kettenreaktion. Diese verläuft zwischen 35 und $50^{\circ} \mathrm{C}$ mit bequem meßbarer Geschwindigkeit und hat einen großen, stark temperaturabhängigen Temperaturkoeffizienten. Wegen der Heterogenität der Reaktion lassen sich keine genauen Angaben über die Zerfallsgeschwindigkeit, unter unseren Bedingungen machen, um so mehr als die Gefäßwände mit Chlorhexoxyd bedeckt sind, also eine Oberfläche vorhanden ist, deren Eigenschaften nicht vorausgesehen werden kann. Man geht aber sicher nicht fehl, anzunehmen, daß bei $40^{\circ} \mathrm{C}$ einige $\%$ des Chlordioxyds zerfallen, bevor sie in das stabile Chlorylfluorid umgesetzt sind. Auf diese Weise wird verständlich, daß bei den bei 22 und $25^{\circ} \mathrm{C}$ ausgeführten Versuchen der Gehalt an freiem Chlor geringer ist als bei den Versuchen bei 40 und $48^{\circ} \mathrm{C}$. Es wird ebenfalls klar, warum bei den Versuchen mit geringem Fluordruck die Menge an freiem Chlor anwächst; denn unter diesem Bedingungen ist die Bildungsgeschwindigkeit des Chlorylfluorids entsprechend kleiner und somit der Eigenzerfall des Chlordioxyds entsprechend größer.

Die Tatsache, daß sich bei tiefen Temperaturen die unbekannte Verbindung $\mathrm{X}$ in größerer Menge vorfindet als bei hohen Temperaturen, deutet an, daß diese Verbindung wenig stabil ist.

\section{Zur Deutung verwandter Reaktionen}

Auf Grund der neu gewonnenen Erkenntnisse lassen sich einige andere Reaktionen, bei denen Chlortrioxyd als Zwischenprodukt auftritt, in einwandfreier Weise deuten und verstehen.

1. Wie schon erwähnt, wurden von Byrns und RoLlefson $\left.\left.{ }^{13}\right)^{30}\right)^{34}$ ) der durch Chlortrioxyd sensibilisierte Ozonzerfall und die photochemische Reaktion zwischen Chlor und Ozon untersucht. In beiden Fällen wurde von ihnen die Bildung von Chlorheptoxyd beobachtet. Sie glaubten auf Grund ihrer Versuche annehmen zu müssen, daß zur Bildung des Heptoxydes die Gegenwart von Ozon notwendig ist.

Der Mechanismus der Heptoxyd-Bildung soll nach ihnen, wie folgt, vor sich gehen:

Chlordioxyd reagiert mit Ozon unter Bildung von angeregtem Chlortrioxyd, dieses reagiert weiter mit Ozon, wobei das $\mathrm{ClO}_{4}$-Radikal entsteht, das sich seinerseits mit einer Trioxydmolekel zum Heptoxyd zusammensetzt.

a.)

b)

c)

$$
\begin{aligned}
\mathrm{ClO}_{2}+\mathrm{O}_{3} & =\mathrm{ClO}_{3}+\mathrm{O}_{2}, \\
\mathrm{ClO}_{3}+\mathrm{O}_{3} & =\mathrm{ClO}_{4}+\mathrm{O}_{2}, \\
\mathrm{ClO}_{4}+\mathrm{ClO}_{3} & =\mathrm{Cl}_{2} \mathrm{O}_{7} .
\end{aligned}
$$

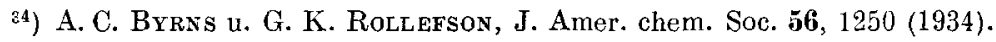


Einen Beweis ihrer Theorie sehen sie in folgendem Versuch: Zu einer größeren Menge von Chlorhexoxyd wird konzentriertes Ozon zugegeben und bei Zimmertemperatur so lange stehen gelassen, bis das Ozon zerfallen ist, dann wird abgekühlt, die Gase werden abgepumpt, es wird von neuem konzentriertes Ozon zugegeben und das Reaktionssystem auf Zimmertemperatur gebracht. Dieses wird mehrere Male wiederholt. Am Ende des Versuches ist alles Chlorhexoxyd verschwunden und an seine Stelle das farblose Chlorheptoxyd getreten.

Auf Grund unserer Versuche läßt sich die nahezu quantitative Umwandlung des Hexoxyds in Heptoxyd in folgender Weise erklären: Das Chlortrioxyd geht nach Maßgabe der von uns festgestellten Daten verschiedene Zerfallsreaktionen ein, die zur Bildung von Chlordioxyd, dem $\mathrm{ClO}_{4}$-Radikal und in geringem Ausmaße von Chlor führen. Die $\mathrm{ClO}_{4}$-Radikale bilden anschließend mit $\mathrm{ClO}_{3}$ Chlorheptoxyd. Alle diese Reaktionen finden ohne Einwirkung des Ozons statt. Das Ozon hat lediglich die wichtige Aufgabe, das gebildete Chlordioxyd sofort wieder in Chlortrioxyd umzuwandeln, und so zu verhindern, daß dieses zerfällt und für die weitere Heptoxydbildung verlorengeht. Die Reaktion zwischen Chlordioxyd und Ozon verläuft, wie SchuMacher ${ }^{18}$ ) festgestellt hat, außerordentlich schnell.

Auf diese Weise wird verständlich, daß sich Chlorhexoxyd bei Gegenwart von Ozon nahezu quantitativ in Heptoxyd umsetzt, ohne daß das Ozon zu der Bildung des Heptoxydes selbst direkt beiträgt. Die bei der Reaktion gebildeten, geringen Chlormengen werden in der Versuchsanordnung von ByRrs und Rollefson durch das bei $-78^{\circ} \mathrm{C}$ durchgeführte Evakuieren entfernt.

2. Der durch Chlor sensibilisierte thermische Ozonzerfall, der von Bodenstein, Schumacher und Mitarbeitern ${ }^{9}{ }^{10}$ ) untersucht wurde, durchläuft zunächst eine Induktionsperiode und folgt dann im großen und ganzen der Geschwindigkeitsgleichung:

Ia

$$
-\frac{\mathrm{d}\left[\mathrm{O}_{3}\right]}{\mathrm{dt}}=\mathrm{k}\left[\mathrm{Cl}_{2}\right]^{1 / 2}\left[\mathrm{O}_{3}\right]^{8 / 2}
$$

Von Schumacher ${ }^{9}$ ) war für den Ablauf der genannten Reaktion der folgende Mechanismus vorgeschlagen worden:

1.

2.

3.

4.

5.

$$
\mathrm{Cl}_{2}+\mathrm{O}_{3}=\mathrm{ClO}+\mathrm{ClO}_{2} \text {, }
$$$$
\mathrm{ClO}_{2}+\mathrm{O}_{3}=\mathrm{ClO}_{3}+\mathrm{O}_{2} \text {, }
$$$$
\mathrm{ClO}_{3}+\mathrm{O}_{3}=\mathrm{ClO}_{2}+2 \mathrm{O}_{2} \text {, }
$$

$$
\mathrm{ClO}_{3}+\mathrm{ClO}_{3}=\mathrm{Cl}_{2}+3 \mathrm{O}_{2}
$$

$$
\mathrm{ClO} \rightarrow 1 / 2 \mathrm{Cl}_{2}+1 / 2 \mathrm{O}_{2} \text {. }
$$


Das Eintreten von Reaktion (1), die die Gesamtreaktion zum Anlaufen bringt, und die eine Aktivierungsenergie von $\mathrm{q}_{1}=26 \mathrm{kcal}$ hat, also relativ langsam ist, kann hierin als gesichert angesehen werden.

Das Auftreten von Reaktion (2) wurde experimentell nachgewiesen. Sie verläuft außerordentlich schnell etwa bei jedem 100. bis 1000. Stoß und ist die Grundlage für die von Schumacher und Stieger entwickelte präparative Herstellung des Hexoxydes.

Die Existenz von Reaktion (3), die für den eigentlichen Ozonzerfall maßgebend ist, wurde von RoLLefson ${ }^{13}$ ), der den durch $\mathrm{ClO}_{3}$ sensibilisierten Ozonzerfall untersuchte, bewiesen. Die genannten Autoren erhielten für diese Reaktion eine Aktivierungsenergie von rund $10,9 \mathrm{kcal}$, die mit dem von SchumaCher berechneten Wert von $11,8 \mathrm{kcal}$ bestens übereinstimmt.

Reaktion (4) stellt den Kettenabbruch dar, während Reaktion (5), die für die Gesamtreaktion ohne Bedeutung ist, lediglich angeben soll, daß das nach (1) gebildete $\mathrm{ClO}$ in Chlor und Sauerstoff umgesetzt wird.

Die $\left[\mathrm{ClO}_{3}\right]$-Konzentration während der Reaktion ist sehr gering; denn beim Ausfrieren des Reaktionsgemisches konnte im allgemeinen kein kondensiertes Hexoxyd festgestellt werden. Lediglich bei mit großen Mengen durchgeführten Versuchen konnten nach dem Durchpumpen der Reaktionsmischung durch ein mit flüssiger Luft gekühltes U-Rohr einige kleine dunkel gefärbte Pünktchen beobachtet werden.

Bodenstein und $\mathrm{SchumaCHER}^{9}$ ) rechneten für die stationäre $\mathrm{ClO}_{3}$-Konzentration bei Gegenwart von etwa $150 \mathrm{~mm} \mathrm{Hg}$ Ozon und $50 \mathrm{~mm} \mathrm{Hg}$ Chlor einen Wert von $2 \cdot 10^{-3} \mathrm{~mm}$ $\mathrm{Hg}$ aus. Da bei diesen Rechnungen für den sterischen Faktor der Reaktion (3) ein solcher von der Größenordnung 1 genommen wurde, sterische Faktoren aber, wie wir heute wissen, oftmals erheblich kleiner sind, so ist es durchaus möglich, daB $\left[\mathrm{ClO}_{3}\right] 10-20 \mathrm{mal}$ größer ist als die früher berechnete, also etwa $5 \cdot 10^{-2} \mathrm{~mm} \mathrm{Hg}$ beträgt. Auf diese Weise wird dann nahezu Übereinstimmung mit der von RoLLEFSoN ${ }^{13}$ ) direkt gemessenen Geschwindigkeit der Reaktion (3) erhalten.

Das soeben diskutierte Schema vermag im großen und ganzen den Reaktionsverlauf dieser Reaktion gut zu erklären, es berücksichtigt aber nicht die Tatsache, daß sich bei Zugabe von Wasserdampf zum Reaktionssystem in geringen Mengen Kristalle von Perchlorsäuremonohydrat an der Gefäßwand niederschlagen und vermag auch nicht den Befund zu erklären, daß bei mit absolut reinen Gasen durchgeführten Versuchen die nach Gleichung Ia berechneten Konstanten gegen Schluß der Reaktion einen deutlichen Abfall zeigen.

Nach unseren neuen Versuchen muß das Schema modifiziert werden; denn wir wissen nunmehr, daß das $\mathrm{ClO}_{3}$ bestimmte Reaktionen eingeht, die in diesem Schema nicht berücksichtigt sind. Wie wir sehen werden, vermag das folgende Schema, das alle die von uns neu gefundenen Reaktionen des $\mathrm{ClO}_{3}$ enthält, die erwähnten bisher noch ungeklärten Befunde zwanglos zu deuten: 
Schema für die thermische Reaktion zwischeil Chlor und Ozon

$$
\begin{aligned}
\mathrm{Cl}_{2}+\mathrm{O}_{3} & =\mathrm{ClO}+\mathrm{ClO}_{2}, \\
\mathrm{ClO}_{2}+\mathrm{O}_{3} & =\mathrm{ClO}_{3}+\mathrm{O}_{2}, \\
\mathrm{ClO}_{3}+\mathrm{O}_{3} & =\mathrm{ClO}_{2}+2 \mathrm{O}_{2}, \\
\mathrm{ClO}_{3}+\mathrm{ClO}_{3} & =2 \mathrm{ClO}_{2}+\mathrm{O}_{2}, \\
\mathrm{ClO}_{3}+\mathrm{ClO}_{3} & =\mathrm{ClO}_{4}+\mathrm{ClO}_{2}, \\
\mathrm{ClO}_{4}+\mathrm{ClO}_{3} & =\mathrm{Cl}_{2} \mathrm{O}_{7}, \\
\mathrm{ClO}_{3}+\mathrm{ClO}_{3} & =\mathrm{Cl}_{2} \quad 3 \mathrm{O}_{2}, \\
\mathrm{ClO} & \rightarrow 1 / 2 \mathrm{Cl}_{2}+1 / 2 \mathrm{O}_{2} .
\end{aligned}
$$

Aus diesem Schema erhält man für die Geschwindigkeit des Ozonzerfalls die folgende Gleichung I

I

$$
-\frac{\mathrm{d}\left[\mathrm{O}_{3}\right]}{\mathrm{dt}}=\mathrm{k}_{1}\left[\mathrm{Cl}_{2}\right]\left[\mathrm{O}_{3}\right]+\mathrm{k}_{2}\left[\mathrm{ClO}_{2}\right]\left[\mathrm{O}_{3}\right]+\mathrm{k}_{3}\left[\mathrm{ClO}_{3}\right]\left[\mathrm{O}_{3}\right]
$$

Den quasistationären Zustand vorausgesetzt, ergeben sich für die Zwischenprodukte folgende Gleichungen:

II

$$
\begin{aligned}
+\frac{\mathrm{d}\left[\mathrm{ClO}_{2}\right]}{\mathrm{dt}} & =\mathrm{k}_{1}\left[\mathrm{Cl}_{2}\right]\left[\mathrm{O}_{3}\right]+\mathrm{k}_{3}\left[\mathrm{ClO}_{3}\right]\left[\mathrm{O}_{3}\right]+\mathrm{k}_{4}\left[\mathrm{ClO}_{3}\right]^{2}+\mathrm{k}_{5}\left[\mathrm{ClO}_{3}\right]^{2} \\
& =\mathrm{k}_{2}\left[\mathrm{ClO}_{2}\right]\left[\mathrm{O}_{3}\right]=-\frac{\mathrm{d}\left[\mathrm{ClO}_{2}\right]}{\mathrm{dt}}
\end{aligned}
$$

III

$$
\begin{aligned}
+\frac{\mathrm{d}\left[\mathrm{ClO}_{3}\right]}{\mathrm{dt}}= & \mathrm{k}_{2}\left[\mathrm{ClO}_{2}\right]\left[\mathrm{O}_{3}\right]=\mathrm{k}_{3}\left[\mathrm{ClO}_{3}\right][\mathrm{O}]_{3}+\mathrm{k}_{4}\left[\mathrm{ClO}_{3}\right]^{2}+\mathrm{k}_{5}\left[\mathrm{ClO}_{3}\right]^{2} \\
& +\mathrm{k}_{6}\left[\mathrm{ClO}_{4}\right]\left[\mathrm{ClO}_{3}\right]+\mathrm{k}_{7}\left[\mathrm{ClO}_{3}\right]^{2}=-\frac{\mathrm{d}\left[\mathrm{ClO}_{3}\right]}{\mathrm{dt}}
\end{aligned}
$$

IV

$$
+\frac{\mathrm{d} \mathrm{ClO}}{4} \cdot=k_{5}\left[\mathrm{ClO}_{3}\right]^{2}=\mathrm{k}_{6}\left[\mathrm{ClO}_{4}\right]\left[\mathrm{ClO}_{3}\right]=-\frac{\mathrm{d}\left[\mathrm{ClO}_{4}\right]}{\mathrm{dt}}
$$

$\mathrm{II}+\mathrm{III}$

$$
\mathrm{k}_{1}\left[\mathrm{Cl}_{2}\right]\left[\mathrm{O}_{3}\right]=\mathrm{k}_{6}\left[\mathrm{ClO}_{4}\right]\left[\mathrm{ClO}_{3}\right]+\mathrm{k}_{7}\left[\mathrm{ClO}_{3}\right]^{2}
$$

und mit IV:

$\mathrm{k}_{1}\left[\mathrm{Cl}_{2}\right]\left[\mathrm{O}_{3}\right]=\left(\mathrm{k}_{5}+\mathrm{k}_{7}\right)\left[\mathrm{ClO}_{3}\right]^{2}$,

hieraus folgt:

V

$$
\left[\mathrm{ClO}_{3}\right]=\sqrt{\frac{\mathrm{k}_{1}\left[\mathrm{Cl}_{3}\right]\left[\mathrm{O}_{3}\right]}{\mathrm{k}_{5}+\mathrm{k}_{7}}}
$$

aus II erhält man:

VI

$$
\left[\mathrm{ClO}_{2}\right]=\frac{\mathrm{k}_{1}\left[\mathrm{Cl}_{2}\right]}{\mathrm{k}_{2}}+\frac{\mathrm{k}_{3}}{\mathrm{k}_{2}}\left[\mathrm{ClO}_{3}\right]+\frac{\mathrm{k}_{4}\left[\mathrm{ClO}_{3}\right]^{2}}{\mathrm{k}_{2}\left[\mathrm{O}_{3}\right]}+\frac{\mathrm{k}_{5}\left[\mathrm{ClO}_{3}\right]^{2}}{\mathrm{k}_{2}\left[\mathrm{O}_{3}\right]}
$$

mit $\mathrm{V}$ ergibt sich:

VII

$$
\begin{aligned}
{\left[\mathrm{ClO}_{2}\right]=} & \frac{\mathrm{k}_{1}}{\mathrm{k}_{2}}\left[\mathrm{Cl}_{2}\right]+\frac{\mathrm{k}_{3}}{\mathrm{k}_{2}}\left(\frac{\mathrm{k}_{\mathrm{L}}}{\mathrm{k}_{5}+\mathrm{k}_{7}}\right)^{1 / 2}\left[\mathrm{Cl}_{2}\right]^{1 / 2}\left[\mathrm{O}_{3}\right]^{1 / 2} \\
& +\frac{\mathrm{k}_{4}}{\mathrm{k}_{2}} \cdot \frac{\mathrm{k}_{1}}{\mathrm{k}_{5}+\mathrm{k}_{7}}\left[\mathrm{Cl}_{2}\right]+\frac{\mathrm{k}_{5}}{\mathrm{k}_{2}} \cdot \frac{\mathrm{k}_{1}}{\mathrm{k}_{5}+\mathrm{k}_{7}}\left[\mathrm{Cl}_{2}\right] \\
= & \left(\frac{\mathrm{k}_{2}}{\mathrm{k}_{2}}+\frac{\mathrm{k}_{1}}{\mathrm{k}_{2}} \cdot \frac{\mathrm{k}_{7}}{\mathrm{k}_{5}}+\frac{\mathrm{k}_{1}}{\mathrm{k}_{7}} \cdot \frac{\mathrm{k}_{5}}{\mathrm{k}_{5}+\mathrm{k}_{7}}\right)\left[\mathrm{Cl}_{2}\right] \\
& +\frac{\mathrm{k}_{3}}{\mathrm{k}_{2}}\left(\frac{\mathrm{k}_{1}}{\mathrm{k}_{5}+\mathrm{k}_{7}}\right)^{1 / 2}\left[\mathrm{Cl}_{2}\right]^{1 / 2}\left[\mathrm{O}_{3}\right]^{1 / 3}
\end{aligned}
$$


Wir wissen aus unseren Versuchen, daß die Aktivierungsenergien der Reaktionen (3), (4) und (5) von der gleichen Größenordnung sind und wissen weiterhin, da $\mathrm{q}_{1}{ }^{9}$ ), um etwa $14 \mathrm{kcal}$ größer ist als diese, also die Geschwindigkeitskonstante von Reaktion (1) um etwa den Faktor $10^{10}$ kleiner ist als die der genannten Reaktionen: Gleichung VII vereinfacht sich infolgedessen zu:

VIII

$$
\left[\mathrm{ClO}_{2}\right] \simeq \frac{\mathrm{k}_{3}}{\mathrm{k}_{2}}\left(\frac{\mathrm{k}_{1}}{\mathrm{k}_{5}+\mathrm{k}_{7}}\right)^{1 / 2}\left[\mathrm{Cl}_{2}\right]^{1 / 2}\left[\mathrm{O}_{3}\right]^{1 / 2}
$$

Mit $V$ und VIII in I eingesetzt erhalten wir als Endgleichung:

IX

$$
-\frac{\mathrm{d}[\mathrm{O}]_{3}}{\mathrm{dt}}=2 \mathrm{k}_{3}\left(\frac{\mathrm{k}_{1}}{\mathrm{k}_{5}+\mathrm{k}_{7}}\right)^{1 / 2}\left[\mathrm{Cl}_{2}\right]^{1 / 2}\left[\mathrm{O}_{3}\right]^{3 / 3}
$$

Setzt man $2 k_{3}\left(\frac{k_{1}}{k_{5}+k_{7}}\right)^{1 / 2}=k$, so geht IX in die experimentell gefundene Gleichung $\mathrm{Ia}-\frac{\mathrm{d}\left[\mathrm{O}_{3}\right]}{\mathrm{dt}}=\mathrm{k}\left[\mathrm{Cl}_{2}\right]^{1 / 2}\left[\mathrm{O}_{3}\right]^{3 / 2}$ über.

In dem neuen Schema verläuft der Kettenabbruch zum erheblichen Teil über Reaktion (6), die zur Bildung von Chlorheptoxyd führt. Das Auftreten von Perchlorsäuremonohydrat bei Gegenwart von Wasserdampf im Reaktionssystem ist somit geklärt. Ebenso wird der Abfall der Konstanten gegen Ende der Reaktion verständlich, der besonders dann merklich wird, wenn man mit hoch gereinigten Gasen arbeitet, so daß das sehr reaktionsfähige Radikal $\mathrm{ClO}_{4}$ nicht durch Verunreinigungen aus dem Reaktionssystem genommen werden kann.

Die Bildung von Chlorheptoxyd geht unter starker Druckverminderung vonstatten. Für $1 \mathrm{~mm}$ gebildetes $\mathrm{Cl}_{2} \mathrm{O}_{7}$ vermindert sich der Druck um $3^{1} / 2 \mathrm{~mm}$. Diese Druckannahme muß durch den Zerfall von $7 \mathrm{~mm}$ Ozon ausgeglichen werden. Da nun bei den früheren Versuchen die Reaktionsgeschwindigkeit durch Druckmessungen verfolgt und der noch vorhandene Ozondruck aus der Gesamtdruckänderung berechnet wurde, wird es verständlich, daß die Konstanten gegen Ende der Reaktion abfallen. Denn um den wirklichen Ozondruck zu erhalten, müßte der nach dem obigen Verfahren berechnete noch um das siebenfache der gebildeten Heptoxydmenge reduziert werden. Der , $\mathrm{Cl}_{2} \mathrm{O}_{7}$-Effekt ${ }^{\prime \prime}$ macht sich in stärkerem Maße nur gegen Ende der Reaktion bemerkbar, da die $\mathrm{Cl}_{2} \mathrm{O}_{7}$ Menge mit der Reaktionszeit anwächst, während die Ozonkonzentration abnimmt, die an dieser anzubringenden Korrektion also in absolutem und relativem Maße zunimmt.

Die Menge des gebildeten Heptoxyds hängt naturgemäß von der Temperatur, dem Chlor- und dem Ozondruck ab. Denn die Konzentration des Trioxyds, die wiederum für die Menge des gebildeten Heptoxyds maßgebend ist, wird durch diese Grölen bestimmt. 
80 Zeitschrift für anorganische und allgemeine Chemie. Band 286. 1956

Man kann somit die experimentellen Ergebnisse der früheren Versuche mit der Annahme, daß sich während der Reaktion Chlorheptoxydmengen bis zur Größenordnung von $1 \mathrm{~mm} \mathrm{Hg}$ bilden, befriedigend erklären.

La Plata (Argentinien), Universidad Nacional de La Plata, Facultad de Quimica y Farmacia, Instituto Superior de Investigaciones.

Bei der Redaktion eingegangen am 9. November 1955. 